\title{
Factores asociados a la sobrevida en pacientes operados por colangiocarcinoma hiliar con intención curativa
}

\author{
Factors associated with survival in patients operated on for hilar cholangiocarcinoma \\ with curative intention
}

Tomás Contreras, Sergio Pacheco, Eduardo Briceño, Jorge Martínez, Juan F. Guerra y Nicolás Jarufe*

Departamento de Cirugía Digestiva, Pontificia Universidad Católica de Chile, Santiago, Chile

\begin{abstract}
Resumen
Objetivo: Determinar la sobrevida a largo plazo y analizar los factores asociados a esta en pacientes operados por colangiocarcinoma hiliar $(\mathrm{CH})$ con intención curativa. Método: Estudio de cohorte no concurrente. Se incluyeron todos los pacientes sometidos a cirugía con intención curativa por CH entre 2002 y 2016. Se realizó un análisis de los factores asociados a la sobrevida mediante Kaplan Meier, test de log-rank y regresión de Cox. Se consideró significativo un valor de $p<0.05$. Resultados: Se operaron 30 pacientes. La mediana de edad fue de 65.5 años (rango: 33-84); 24 (80\%) fueron de sexo masculino. El margen quirúrgico resultó negativo en 27 (90\%) pacientes. Veintiún (70\%) pacientes presentaron complicaciones y 3 (10\%) fallecieron en el posoperatorio. Las sobrevidas al año, a 5 años y a 10 años fueron del $65.7 \%$, el 37.3\% y el 16.6\%, respectivamente. En el análisis multivariable, el único factor asociado a la sobrevida fue el estadio T (hazard ratio: 0.309; intervalo de confianza del 95\%: 0.101-0.942; $p=0.03$ ). Discusión: Los pacientes operados por $\mathrm{CH}$ con intención curativa en nuestro centro presentan una adecuada sobrevida a largo plazo, con una elevada morbimortalidad posoperatoria. El único factor que se asoció a la sobrevida fue el estadio $T$.
\end{abstract}

PALABRAS CLAVE: Cirugía. Sobrevida. Tumor de Klatskin.

\begin{abstract}
Objective: To determine the long-term survival and to analyze the factors associated with it in the patients operated on for hilar cholangiocarcinoma $(\mathrm{HC})$ with curative intention. Method: Non concurrent cohort study. We included all patients who underwent surgery with curative intent for HC between 2002 and 2016. An analysis of factors associated with survival using Kaplan Meier, log-rank test and Cox regression was performed. A p-value less than 0.05 was considered significant. Results: Thirty patients were operated on. The median age was 65.5 years (range: 33-84); 24 patients (80\%) were male. The surgical margin was negative in 27 patients (90\%). Twenty-one patients (70\%) presented complications and three patients (10\%) died postoperatively. Survival at the year, 5 years and 10 years were $65.7 \%, 37.3 \%$ and $16.6 \%$, respectively. In multivariable analysis, the only factor associated with survival was the T stage (hazard ratio: $0.309 ; 95 \%$ confidence interval: 0.101 0.942; $p=0.03$ ). Discussion: Patients operated on for $\mathrm{HC}$ with curative intent in our center have adequate long-term survival, with high postoperative morbidity and mortality. The only factor that was associated with survival was $T$ stage.
\end{abstract}

KEY WORDS: Surgery. Survival. Klatskin tumor.

\footnotetext{
Correspondencia:

*Nicolás Jarufe-Cassis

Diagonal Paraguay, 362, Piso 4

CP 83330077, Santiago, Chile

E-mail: njarufe@med.puc.cl
}

Fecha de recepción: 15-07-2018

Fecha de aceptación: 06-04-2019

DOI:10.24875/CIRU.19000603
Cir Cir. 2019;87:508-515

Contents available at PubMed www.cirugiaycirujanos.com 


\section{Introducción}

El colangiocarcinoma es un tumor maligno originado en el epitelio de los conductos biliares. Es un tumor infrecuente, que representa alrededor de un $2 \%$ de los tumores malignos en el ser humano ${ }^{1}$, pero es la segunda neoplasia maligna más frecuente del hígado².

De acuerdo con su ubicación, los colangiocarcinomas se han clasificado como intrahepáticos, hiliares (también conocidos como tumores de Klatskin) y distales. Los colangiocarcinomas hiliares $(\mathrm{CH})$ son los más frecuentes y representan aproximadamente un $50-70 \%$ de todos los colangiocarcinomas ${ }^{3,4}$.

El único tratamiento curativo actualmente disponible es la cirugía con márgenes negativos ${ }^{3,5}$. En este grupo de pacientes, la mediana de sobrevida global varía entre 19 y 39 meses $^{6}$. Sin embargo, la cirugía resectiva se asocia a una elevada morbimortalidad ${ }^{7}$. Sin tratamiento quirúrgico, su pronóstico suele ser malo, con una sobrevida promedio menor de 1 año $0^{8-10}$.

Los factores previamente reportados que determinan la sobrevida a largo plazo son el estadio $\mathrm{T}$, el compromiso linfonodal, el margen quirúrgico positivo y la diferenciación tumoral ${ }^{6}$.

El objetivo del presente estudio es determinar la sobrevida a largo plazo y analizar los factores asociados a esta en los pacientes operados por $\mathrm{CH}$ con intención curativa.

\section{Método}

\section{Pacientes}

Estudio de cohorte no concurrente. Se incluyeron todos los pacientes sometidos a cirugía con intención curativa por $\mathrm{CH}$ en el Hospital Clínico de la Pontificia Universidad Católica de Chile entre enero de 2002 y diciembre de 2016. Los criterios de exclusión fueron: pacientes sin indicación quirúrgica y pacientes sometidos a cirugías no resectivas (laparotomía o laparoscopía exploradora) o paliativas.

Los criterios de irresecabilidad fueron: pacientes con enfermedad en etapa IV, enfermos con adenopatías metastásicas alejadas del pedículo hepático, pacientes con extensión intrahepática del tumor sin una zona segura para realizar la anastomosis biliodigestiva, pacientes en los cuales fuese factible realizar una cirugía con márgenes negativos pero que el futuro remanente hepático fuese insuficiente para tolerar la cirugía (pese a procedimientos como la embolización portal preoperatoria), tumores que comprometían la confluencia biliar y se extendían por los ductos biliares segmentarios de forma bilateral, tumores con extensión unilateral en la vía biliar intrahepática y compromiso vascular unilateral con atrofia del lóbulo contralateral.

\section{Manejo preoperatorio y cirugía}

En el preoperatorio se realizó el diagnóstico de $\mathrm{CH}$ en pacientes con un tumor de aspecto maligno en los estudios de imagen (tomografía computada, resonancia magnética y en algunos casos también tomografía por emisión de positrones) en el conducto hepático común próximo a la confluencia, en la confluencia de los conductos hepáticos o en la confluencia, y que afectara los conductos hepáticos izquierdo o derecho. No se realizó biopsia sistemática para certificar el diagnóstico de $\mathrm{CH}$. El drenaje preoperatorio de la vía biliar se realizó según decisión del cirujano tratante, tras el análisis de cada caso en particular. En general, el drenaje se indicó en pacientes con bilirrubina > $10 \mathrm{mg} / \mathrm{dl}$. No se realizó embolización portal preoperatoria en ningún paciente, dado que los futuros remanentes hepáticos fueron considerados suficientes. La ubicación del tumor se estratificó según la clasificación de Bismuth-Corlette ${ }^{11}$.

Se comenzó la cirugía mediante una laparotomía exploradora para descartar carcinomatosis peritoneal, metástasis y extensión de la enfermedad más allá del ligamento hepatoduodenal. Posteriormente se realizó la resección de la vía biliar asociada a una hepatectomía en la mayoría de los pacientes, linfadenectomía completa del ligamento hepatoduodenal y, en caso necesario, resección vascular más su respectiva reconstrucción. En algunos pacientes se realizó una maniobra de Pringle intermitente según decisión del cirujano.

Se realizó una biopsia intraoperatoria para descartar neoplasia en los márgenes de sección de la vía biliar. Cuando el margen fue positivo, se realizó una nueva resección hasta alcanzar márgenes microscópicos negativos (R0).

\section{Registro, seguimiento y análisis estadístico}

Se revisaron las fichas clínicas y los informes de anatomía patológica. Se diseñó una base de datos en el software Microsoft Excel para el registro de datos demográficos y clínicos, informes de anatomía patológica, 
morbimortalidad posoperatoria (definida hasta los 90 días posteriores a la cirugía) y sobrevida de los pacientes en el seguimiento.

Los resultados de la biopsia diferida se consignaron según la clasificación TNM (Union for International Cancer Control/American Joint Committee on Cancer, $7^{\text {th }}$ edition). Las complicaciones posoperatorias se estratificaron según la clasificación de Clavien-Dindo ${ }^{12}$, considerando como mayores aquellas $\geq$ III. La insuficiencia hepática posoperatoria se definió como aquella de grado B o mayor según el International Study Group of Liver Surgery ${ }^{13}$.

Los pacientes fueron seguidos tras la cirugía de forma ambulatoria cada 3 meses durante los primeros 2 años con exploración física, exámenes de laboratorio (incluyendo pruebas hepáticas y marcador tumoral CA 19-9) y tomografía computada de tórax, abdomen y pelvis. Tras este periodo, los controles fueron cada 6 meses hasta los 5 años, para posteriormente realizar controles anuales.

Para la descripción de variables continuas se utilizaron los estadígrafos mediana y rango. La descripción de las variables cualitativas se realizó mediante frecuencias absolutas y porcentajes. Se realizó un análisis univariable de los factores asociados a la sobrevida mediante el método de Kaplan Meier, comparando entre los grupos mediante test de log-rank. Aquellos factores que en el análisis univariable presentaron un valor de $p<0.05$ fueron ingresados a un análisis multivariable mediante regresión de Cox para modelar los riesgos que afectaban la supervivencia de la población en estudio. Se utilizó el programa SPSS 22.0 (SPSS Inc., Chicago, IL, EE.UU.) para el análisis estadístico, considerando significativo un valor de $p<0.05$.

\section{Ética}

Este estudio se realizó de acuerdo con las normas éticas de la Pontificia Universidad Católica de Chile. No se requirió consentimiento informado específico debido a la naturaleza anónima de los datos utilizados. Ningún dato obtenido permitió la identificación de los pacientes.

\section{Resultados}

Durante el periodo en estudio se operaron 30 pacientes por $\mathrm{CH}$ con intención curativa. La mediana de edad fue de 65.5 años (rango: 33-84); 24 (80\%) pacientes fueron de sexo masculino. Las restantes variables demográficas se detallan en la tabla 1.
Tabla 1. Variables demográficas y clínicas de los pacientes en estudio

\begin{tabular}{lc}
\hline Variables & $\mathbf{N}=\mathbf{3 0}$ \\
\hline $\begin{array}{l}\text { Sexo } \\
\text { - Masculino }\end{array}$ & $24(80 \%)$ \\
- Femenino & $6(20 \%)$ \\
Edad & $65.5(33-84)$ \\
Colangitis preoperatoria & $5(16.7 \%)$ \\
CA 19-9 (U/ml) & $240(1.4-1580)$ \\
Bilirrubina preoperatoria (mg/dl) & $5.9(0.7-24.6)$ \\
Drenaje preoperatorio de la vía biliar & $10(33.3 \%)$ \\
- Mediante colangiopancreatografía retrógrada & $4(13.3 \%)$ \\
$\quad$ endoscópica & \\
- Mediante punción transparietohepática & $6(20 \%)$ \\
\hline
\end{tabular}

De acuerdo con la clasificación de Bismuth-Colette, Ios $\mathrm{CH}$ más frecuentes fueron los de tipo III $\mathrm{B}$, en 12 pacientes $(40 \%)$, seguidos por los de tipo III A, en 8 enfermos $(26,7 \%)$, como se detalla en la tabla 2 . La cirugía más frecuentemente realizada fue la hepatectomía izquierda más resección de la vía biliar, en 13 casos (43.3\%); fue necesario realizar una resección vascular en 5 (16.7\%) pacientes.

El margen quirúrgico resultó negativo en 27 (90\%) pacientes. Seis $(20 \%)$ pacientes presentaron compromiso ganglionar en la biopsia operatoria. El estadio TNM más frecuente fue el II, en 20 enfermos (66.7\%), como se especifica en la tabla 3.

Dos $(6.6 \%)$ pacientes presentaron lesiones vasculares en el intraoperatorio. Uno de ellos tuvo una lesión de la vena cava inferior y el otro una lesión de la vena suprahepática izquierda. En ambos casos, las lesiones se repararon mediante sutura, logrando una adecuada hemostasia. No se presentaron complicaciones posteriores relacionadas con estas lesiones.

Veintiún $(70 \%)$ pacientes presentaron complicaciones en el posoperatorio; la más frecuente fue la colección intraabdominal, en 7 (23.3\%) pacientes, como se detalla en la tabla 4. Las complicaciones clasificadas como Clavien-Dindo $\geq$ III se presentaron en $10(33.3 \%)$ pacientes. Ocho (26.7\%) pacientes requirieron reoperación. Fallecieron $3(10 \%)$ pacientes en el periodo posoperatorio: dos debido a una trombosis portal y el otro por un shock séptico secundario a una colección intraabdominal.

Se realizó quimioterapia y radioterapia adyuvante en $7(23.3 \%)$ y $5(16.7 \%)$ pacientes, respectivamente. Las sobrevidas al año, a los 5 años y a los 10 años fueron del $65.7 \%$, el $37.3 \%$ y el $16.6 \%$, respectivamente (Fig. 1). Once (36.7\%) pacientes presentaron 
Tabla 2. Variables quirúrgicas

\begin{tabular}{lc}
\hline Variables & N = 30 \\
\hline Clasificación de Bismuth-Corlette & \\
- II & $4(13.3 \%)$ \\
- IIIA & $8(26.7 \%)$ \\
- IIIB & $12(40 \%)$ \\
- IV & $6(20 \%)$ \\
Cirugía efectuada & \\
- Hepatectomía izquierda más resección de & $13(43.3 \%)$ \\
vía biliar & $7(23.3 \%)$ \\
- Hepatectomía derecha extendida más & $4(13.3 \%)$ \\
resección de vía biliar & $3(10 \%)$ \\
- Hepatectomía derecha más resección de & $3(10 \%)$ \\
vía biliar & \\
- Hepatectomía izquierda extendida más & \\
resección de vía biliar & \\
- Resección de vía biliar aislada & \\
Resección vascular & $5(16.7 \%)$ \\
- Resección de vena porta & $4(13.3 \%)$ \\
- Resección parcial de vena cava inferior & $1(3.3 \%)$ \\
Reconstrucción portal & \\
- Puente venoso & \\
- Prótesis vascular de politetrafluoroetileno & $2(6.6 \%)$ \\
Tiempo operatorio (minutos) & $2(6.6 \%)$ \\
Sangrado intraoperatorio estimado (ml) & $360(240-720)$ \\
Glóbulos rojos transfundidos (unidades) & $1500(120-2000)$ \\
Maniobra de Pringle & $2(0-8)$ \\
Tiempo total de maniobra de Pringle (minutos) & $45(20-90)$ \\
\hline
\end{tabular}

recidiva en el seguimiento, con una mediana de tiempo de 15 meses (rango: 3-48) desde la cirugía original. De estos pacientes, $5(45.5 \%)$ presentaron recidiva en el hígado, $5(45.5 \%)$ en la vía biliar y $1(9 \%)$ en el pulmón.

La tabla 5 muestra el análisis univariable de los factores asociados a la sobrevida. Las variables que resultaron estadísticamente significativas fueron los estadios $T(p=0.01)$ (Fig. 2$)$ y $N(p=0.03)$ (Fig. 3). En la regresión de Cox (Tabla 6), la única variable asociada a la sobrevida fue el estadio T (hazard ratio: 0.309; intervalo de confianza del 95\%: 0.101-0.942; $p=0.03$ ).

\section{Discusión}

En el presente estudio, la sobrevida a largo plazo es similar a la reportada en series previas con mayor tamaño muestral ${ }^{4,14,15}$. En el análisis multivariable, el único factor asociado a la sobrevida fue el estadio $\mathrm{T}$.

Las características clínicas y demográficas de los pacientes analizados son similares a las reportadas en estudios previos ${ }^{6,16}$. El drenaje preoperatorio de la vía biliar se realizó según decisión de cada cirujano
Tabla 3. Variables histopatológicas

\begin{tabular}{|c|c|}
\hline Variables & $N=30$ \\
\hline Diámetro tumoral máximo (cm) & $3(1.2-6)$ \\
\hline $\begin{array}{l}\text { Diferenciación } \\
\text { - Bien diferenciado } \\
\text { - Moderadamente diferenciado } \\
\text { - Mal diferenciado }\end{array}$ & $\begin{array}{l}18(60 \%) \\
8(26.7 \%) \\
4(13.3 \%)\end{array}$ \\
\hline Infiltración linfovascular & $15(50 \%)$ \\
\hline Infiltración perineural & $3(10 \%)$ \\
\hline $\begin{array}{l}\text { Margen quirúrgico } \\
\text { - Negativo } \\
\text { - Positivo }\end{array}$ & $\begin{array}{c}27(90 \%) \\
3(10 \%)\end{array}$ \\
\hline $\begin{array}{l}\text { Estadio } T^{*} \\
- \text { - T2a } \\
-\mathrm{T} 2 \mathrm{~b} \\
-\mathrm{T} 3 \\
-\mathrm{T} 4\end{array}$ & $\begin{array}{c}15(50 \%) \\
8(26.7 \%) \\
5(16.7 \%) \\
26.7 \%)\end{array}$ \\
\hline $\begin{array}{l}\text { Estadio } N^{*} \\
-\mathrm{NO} \\
-\mathrm{N} 1\end{array}$ & $\begin{array}{c}24(80 \%) \\
6(20 \%)\end{array}$ \\
\hline $\mathrm{MO}^{\star}$ & $30(100 \%)$ \\
\hline $\begin{array}{l}\text { Estadio TNM } \\
- \text { II } \\
- \text { III } \\
- \text { IV }\end{array}$ & $\begin{array}{c}20(66.7 \%) \\
8(26.7 \%) \\
2(6.7 \%)\end{array}$ \\
\hline
\end{tabular}

tratante, y se utilizó en un $33.3 \%$ de los pacientes, lo cual difiere de otras series, en las que generalmente se realiza en la mayoría de los enfermos ${ }^{6,7,16}$. La utilidad real del drenaje preoperatorio de la vía biliar es aún materia de debate. La ictericia obstructiva se asocia a colangitis, disfunción renal y hepática ${ }^{17,18}$, pero el drenaje preoperatorio se asocia a colangitis, mayor estadía hospitalaria y, en casos excepcionales, metástasis en el trayecto del drenaje transparietohepático ${ }^{19,20}$. Un metaanálisis no demostró mayor beneficio con el drenaje preoperatorio de la vía biliar en pacientes con $\mathrm{CH}^{21}$. Una indicación absoluta del drenaje preoperatorio de la vía biliar es previo a la realización de una embolización portal, debido a que la ictericia reduce la hipertrofia hepática que se desea obtener $^{21}$. En el presente estudio no se realizó embolización portal antes de la cirugía en ningún paciente, debido a que los futuros remanentes hepáticos se consideraron suficientes en los estudios de imagen preoperatorios.

La mayoría de los pacientes (86.7\%) presentaban un $\mathrm{CH}$ clasificado como III o IV según la clasificación de Bismuth-Corlette ${ }^{11}$, por lo cual requirieron cirugías que combinaban resección de la vía biliar más 
Tabla 4. Morbimortalidad posoperatoria

\begin{tabular}{lc}
\hline Variables & $\mathbf{N}=\mathbf{3 0}$ \\
\hline Complicaciones posoperatorias & $21(70 \%)$ \\
- Colección intraabdominal & $7(23.3 \%)$ \\
- Fístula biliar externa & $6(20 \%)$ \\
- Trombosis portal & $4(13.3 \%)$ \\
- Insuficiencia hepática & $3(10 \%)$ \\
- Hemoperitoneo & $2(6.7 \%)$ \\
- Biliperitoneo & $1(3.3 \%)$ \\
- Colangitis & $1(3.3 \%)$ \\
- Hemorragia digestiva baja & $1(3.3 \%)$ \\
- Hepatitis isquémica & $1(3.3 \%)$ \\
Clasificación de Clavien-Dindo & \\
- I & $2(6.7 \%)$ \\
- II & $9(30 \%)$ \\
- Illa & $2(6.7 \%)$ \\
- Illb & $4(13.3 \%)$ \\
- IVa & $1(3.3 \%)$ \\
- V & $3(10 \%)$ \\
Reoperación & $8(26.7 \%)$ \\
Trombectomía portal & $2(6.7 \%)$ \\
Drenaje percutáneo guiado por tomografía computada & $2(6.7 \%)$ \\
Estadía posoperatoria (días) & $13.5(5-60)$ \\
Mortalidad operatoria & $3(10 \%)$ \\
Reingreso hospitalario & $6(20 \%)$ \\
\hline
\end{tabular}

hepatectomía. En solo 3 (10\%) pacientes se realizó una resección aislada de la vía biliar; de ellos, dos pacientes resultaron con márgenes quirúrgicos negativos. Existe evidencia de que la hepatectomía asociada a la resección de la vía biliar, independientemente del estadio, permite una mayor tasa de cirugía R0 que la resección aislada de la vía biliar22-26.

En cuanto a la resección vascular, la mayoría de los estudios señalan que no se asocia a una disminución significativa de la sobrevida a largo plazo ${ }^{27-30}$. En el presente estudio, solo cinco pacientes requirieron una resección vascular, lo cual no modificó su posterior sobrevida.

Veintisiete $(90 \%)$ pacientes resultaron con márgenes quirúrgicos negativos, lo que supone un porcentaje mayor que los reportados en estudios previos ${ }^{6,714-16}$. Esto podría deberse a la rigurosa selección de los pacientes en los estudios de imagen preoperatorios y a la continua evaluación de los enfermos en comités oncológicos multidisciplinarios.

Se presentaron complicaciones en 21 (70\%) pacientes; aquellas calificadas como mayores ocurrieron en $10(33.3 \%)$, lo cual es comparable con lo reportado en estudios previos, en los que la morbilidad posoperatoria varía entre el $14 \%$ y el $72 \%^{29,31-33}$. La

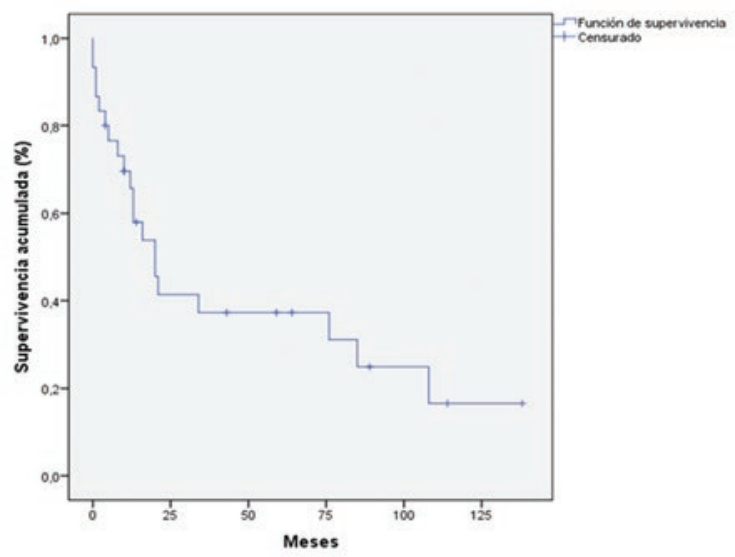

Figura 1. Sobrevida global de los pacientes en estudio.

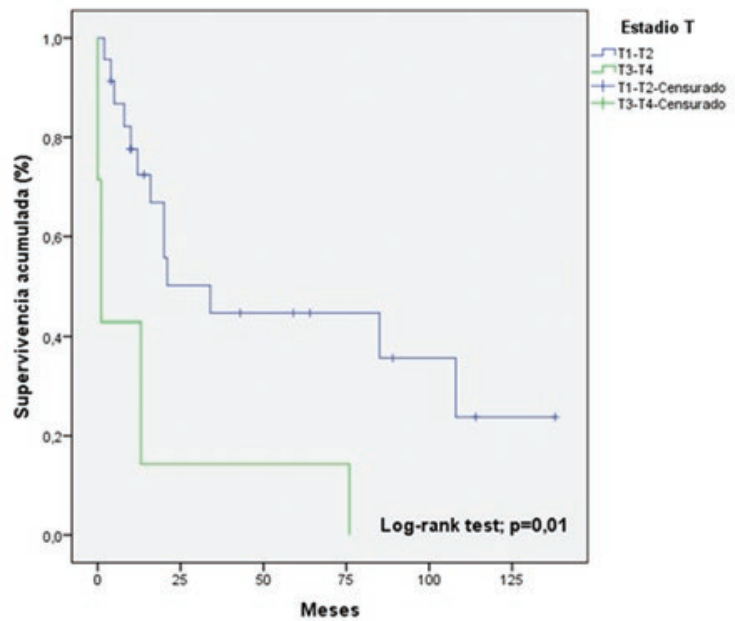

Figura 2. Sobrevida de los pacientes según el estadio $T$.

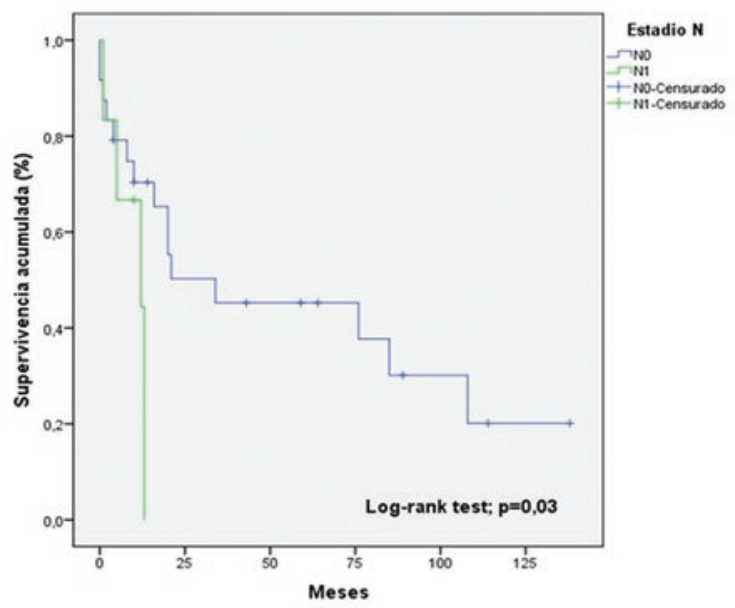

Figura 3. Sobrevida de los pacientes según el estadio N.

mortalidad operatoria se presentó en $3(10 \%)$ pacientes, lo cual se encuentra dentro del rango de otras publicaciones, en las que va del $0 \%$ al $15 \%^{34-37}$. 
Tabla 5. Análisis univariable de factores asociados a la sobrevida

\begin{tabular}{|c|c|c|c|c|}
\hline Variable & Categoría & $N=30$ & Sobrevida (meses) & $\mathrm{p}$ \\
\hline \multirow[t]{2}{*}{$\overline{\text { Edad }}$} & $<65$ años & $13(43.3 \%)$ & $59.1 \pm 17.4$ & 0.87 \\
\hline & $\geq 65$ años & $17(56.7 \%)$ & $45.8 \pm 12.8$ & \\
\hline \multirow[t]{2}{*}{ Sexo } & Masculino & $24(80 \%)$ & $50.8 \pm 11.2$ & 0.97 \\
\hline & Femenino & $6(20 \%)$ & $54.8 \pm 24.1$ & \\
\hline \multirow[t]{2}{*}{ Colangitis preoperatoria } & Sí & $5(16.7 \%)$ & $20.4 \pm 9.1$ & 0.34 \\
\hline & No & $25(83.3 \%)$ & $62.3 \pm 13$ & \\
\hline \multirow[t]{2}{*}{ CA 19-9 } & $<240 \mathrm{U} / \mathrm{ml}$ & $14(46.7 \%)$ & $55.8 \pm 16.6$ & 0.94 \\
\hline & $\geq 240 \mathrm{U} / \mathrm{ml}$ & $16(53.3 \%)$ & $48.5 \pm 13.4$ & \\
\hline \multirow[t]{2}{*}{ Bilirrubina preoperatoria } & $<2 \mathrm{mg} / \mathrm{dl}$ & $9(30 \%)$ & $93.5 \pm 20.8$ & 0.08 \\
\hline & $\geq 2 \mathrm{mg} / \mathrm{dl}$ & $21(70 \%)$ & $36.8 \pm 10.7$ & \\
\hline \multirow[t]{2}{*}{ Drenaje preoperatorio de vía biliar } & Sí & $10(33.3 \%)$ & $25.7 \pm 10.7$ & 0.26 \\
\hline & No & $20(66.7 \%)$ & $65.7 \pm 14.3$ & \\
\hline \multirow[t]{2}{*}{ Bismuth-Corlette } & $|-| \mid$ & $4(13.3 \%)$ & $58.3 \pm 12.8$ & 0.77 \\
\hline & III-IV & $26(86.7 \%)$ & $42.5 \pm 21$ & \\
\hline \multirow[t]{2}{*}{ Resección vascular } & No & $25(83.3 \%)$ & $64.1 \pm 13.4$ & 0.16 \\
\hline & Sí & $5(16.7 \%)$ & $13.8 \pm 7$ & \\
\hline \multirow[t]{2}{*}{ Tiempo operatorio } & $<360 \min$ & $17(56.7 \%)$ & $66.3 \pm 16.6$ & 0.19 \\
\hline & $\geq 360 \mathrm{~min}$ & $13(43.3 \%)$ & $39.3 \pm 13.9$ & \\
\hline \multirow[t]{2}{*}{ Transfusiones de glóbulos rojos } & No & $19(63.3 \%)$ & $81.1 \pm 18.9$ & 0.13 \\
\hline & Sí & $11(36.7 \%)$ & $29.2 \pm 8.9$ & \\
\hline \multirow[t]{2}{*}{ Maniobra de Pringle } & Sí & $8(26.7 \%)$ & $45.8 \pm 18.7$ & 0.56 \\
\hline & No & $22(73.3 \%)$ & $58.4 \pm 14.1$ & \\
\hline \multirow[t]{2}{*}{ Diámetro tumoral } & $<3 \mathrm{~cm}$ & $14(46.7 \%)$ & $72 \pm 14.8$ & 0.09 \\
\hline & $\geq 3 \mathrm{~cm}$ & $16(53.3 \%)$ & $39.1 \pm 13.6$ & \\
\hline \multirow[t]{2}{*}{ Diferenciación } & Bien diferenciado & $18(60 \%)$ & $65.3 \pm 15.1$ & 0.42 \\
\hline & Moderadamente y mal diferenciado & $12(40 \%)$ & $28.8 \pm 11$ & \\
\hline \multirow[t]{2}{*}{ Infiltración linfovascular } & No & $15(50 \%)$ & $72.6 \pm 17.2$ & 0.17 \\
\hline & Sí & $15(50 \%)$ & $37.8 \pm 12.2$ & \\
\hline \multirow[t]{2}{*}{ Infiltración perineural } & No & $3(10 \%)$ & $51.3 \pm 20.1$ & 0.42 \\
\hline & Sí & $27(90 \%)$ & $53.7 \pm 12.5$ & \\
\hline \multirow[t]{2}{*}{ Márgenes } & Negativos & $27(90 \%)$ & $61.6 \pm 12.6$ & 0.33 \\
\hline & Positivos & $3(10 \%)$ & $10.6 \pm 1.5$ & \\
\hline \multirow[t]{2}{*}{ Estadio $T^{*}$} & $\mathrm{~T} 1-\mathrm{T} 2$ & $23(76.7 \%)$ & $69.3 \pm 14$ & 0.01 \\
\hline & T3-T4 & $7(23.3 \%)$ & $14.8 \pm 9.6$ & \\
\hline \multirow[t]{2}{*}{ Estadio $N^{*}$} & NO & $24(80 \%)$ & $69 \pm 13.6$ & 0.03 \\
\hline & N1 & $6(20 \%)$ & $9.4 \pm 2.1$ & \\
\hline \multirow[t]{2}{*}{ Quimioterapia adyuvante } & Sí & $7(23.3 \%)$ & $56.1 \pm 19$ & 0.63 \\
\hline & No & $23(76.7 \%)$ & $56.3 \pm 14$ & \\
\hline \multirow[t]{2}{*}{ Radioterapia adyuvante } & Sí & $5(16.7 \%)$ & $51.6 \pm 22.8$ & 0.95 \\
\hline & No & $25(83.3 \%)$ & $58 \pm 13.3$ & \\
\hline
\end{tabular}


Tabla 6. Análisis multivariable de factores asociados a la sobrevida

\begin{tabular}{|c|c|c|c|c|}
\hline Variable & Categoría & $\begin{array}{l}\text { Hazard } \\
\text { ratio }\end{array}$ & $\begin{array}{c}\text { Intervalo de } \\
\text { confianza del } 95 \%\end{array}$ & $p$ \\
\hline Estadio $T^{*}$ & $\begin{array}{l}\text { T1-T2 } \\
\text { T3-T4 }\end{array}$ & 0.309 & $0.101-0.942$ & 0.03 \\
\hline Estadio $\mathrm{N}^{*}$ & $\begin{array}{l}\text { No } \\
\text { N1 }\end{array}$ & 0.591 & $0.159-2.199$ & 0.43 \\
\hline
\end{tabular}

${ }^{*}$ Clasificado de acuerdo con la Union for International Cancer Control/American Joint Committee on Cancer, $7^{\text {th }}$ edition.

Cabe destacar que, a diferencia de otros estudios, no se encontró asociación entre la sobrevida y los márgenes negativos, la diferenciación tumoral y la invasión perineural. Esto podría deberse a la cantidad relativamente baja de pacientes en estudio, lo cual aumenta la probabilidad de presentar un error de tipo II en la metodología de la presente investigación.

Las limitaciones de este estudio son su carácter observacional, unicéntrico y retrospectivo, y el ya mencionado pequeño tamaño muestral si se compara con otras series publicadas previamente $e^{7,16,38}$.

\section{Conclusiones}

Los pacientes operados por $\mathrm{CH}$ con intención curativa en nuestro centro presentan una adecuada sobrevida a largo plazo, con una elevada morbimortalidad asociada, pero que es comparable a la de estudios previos. En el análisis multivariable, el único factor que se asoció a la sobrevida fue el estadio $\mathrm{T}$.

La cirugía de los $\mathrm{CH}$ requiere habitualmente grandes resecciones hepáticas, incluido el segmento caudado, junto con la totalidad de la vía biliar afectada y la posterior reconstrucción; es decir, es una cirugía de alta demanda técnica que se realiza en pacientes que a veces no se encuentran en un adecuado estado nutricional y general, por ictericia e infecciones asociadas. Esto explica las altas tasas de morbimortalidad en este trabajo y en la literatura internacional. Por ello, debe ser realizada por cirujanos de experiencia y en centros con recursos adecuados.

\section{Conflicto de intereses}

Los autores no refieren conflicto de intereses.

\section{Financiamiento}

La presente investigación no posee fuente de financiamiento.

\section{Responsabilidades éticas}

Protección de personas y animales. Los autores declaran que para esta investigación no se han realizado experimentos en seres humanos ni en animales.

Confidencialidad de los datos. Los autores declaran que han seguido los protocolos de su centro de trabajo sobre la publicación de datos de pacientes.

Derecho a la privacidad y consentimiento informado. Los autores han obtenido el consentimiento informado de los pacientes y/o sujetos referidos en el artículo. Este documento obra en poder del autor de correspondencia.

\section{Bibliografía}

1. Soares KC, Kamel I, Cosgrove DP, Herman JM, Pawlik TM. Hilar cholangiocarcinoma: diagnosis, treatment options, and management. Hepatobiliary Surg Nutr. 2014;3:18-34.

2. Ramia JM. Hilar cholangiocarcinoma. World J Gastrointest Oncol. 2013; 5:113-4.

3. Bhardwaj N, Garcea G, Dennison AR, Maddern GJ. The surgical management of Klatskin tumours: has anything changed in the last decade? World J Surg. 2015;39:2748-56.

4. DeOliveira ML, Cunningham SC, Cameron JL, Kamangar F, Winter JM, Lillemoe KD, et al. Cholangiocarcinoma. Ann Surg. 2007;245:755-62.

5. Zaydfudim VM, Rosen CB, Nagorney DM. Hilar cholangiocarcinoma. Surg Oncol Clin N Am. 2014;23:247-63.

6. Groot Koerkamp B, Wiggers JK, Gonen M, Doussot A, Allen PJ, Besselink $\mathrm{MGH}$, et al. Survival after resection of perihilar cholangiocarcinoma - development and external validation of a prognostic nomogram. Ann Oncol. 2015;26:1930-5.

7. Wiggers JK, Groot Koerkamp B, Cieslak KP, Doussot A, van Klaveren D, Allen PJ, et al. Postoperative mortality after liver resection for perihilar cholangiocarcinoma: development of a risk score and importance of biliary drainage of the future liver remnant. J Am Coll Surg. 2016;223:321-31.e1.

8. Strasberg SM. Resection of hilar cholangiocarcinoma. HPB Surg. 1998; 10:415-8.

9. Saxena A, Chua TC, Chu FC, Morris DL. Improved outcomes after aggressive surgical resection of hilar cholangiocarcinoma: a critical analysis of recurrence and survival. Am J Surg. 2011;202:310-20.

10. Brown KM, Geller DA. Proximal biliary tumors. Surg Clin North Am. 2014;94:311-23.

11. Bismuth $\mathrm{H}$, Corlette MB. Intrahepatic cholangioenteric anastomosis in carcinoma of the hilus of the liver. Surg Gynecol Obstet. 1975;140:170-8.

12. Dindo D, Demartines N, Clavien PA. Classification of surgical complications. Ann Surg. 2004;240:205-13.

13. Rahbari NN, Garden OJ, Padbury R, Brooke-Smith M, Crawford M, Adam R, et al. Posthepatectomy liver failure: a definition and grading by the International Study Group of Liver Surgery (ISGLS). Surgery. 2011; 149:713-24

14. Hasegawa S, Ikai I, Fujii H, Hatano E, Shimahara Y. Surgical resection of hilar cholangiocarcinoma: analysis of survival and postoperative complications. World J Surg. 2007;31:1256-63.

15. Yubin L, Chihua F, Zhixiang J, Jinrui O, Zixian L, Jianghua Z, et al. Surgical management and prognostic factors of hilar cholangiocarcinoma: experience with 115 cases in China. Ann Surg Oncol. 2008;15:2113-9.

16. Groot Koerkamp B, Wiggers JK, Allen PJ, Besselink MG, Blumgart LH, Busch ORC, et al. Recurrence rate and pattern of perihilar cholangiocarcinoma after curative intent resection. J Am Coll Surg. 2015; 221:1041-9.

17. Rea DJ, Muñoz-Juárez M, Farnell MB, Donohue JH, Que FG, Crownhart B, et al. Major hepatic resection for hilar cholangiocarcinoma. Arch Surg. 2004;139:514.

18. Lai EC, Mok FP, Fan ST, Lo CM, Chu KM, Liu CL, et al. Preoperative endoscopic drainage for malignant obstructive jaundice. $\mathrm{Br} J$ Surg. 1994:81:1195-8.

19. Laurent $A$, Tayar $C$, Cherqui D. Cholangiocarcinoma: preoperative biliary drainage (Con). HPB (Oxford). 2008;10:126-9.

20. Sakata J, Shirai Y, Wakai T, Nomura T, Sakata E, Hatakeyama K. Catheter tract implantation metastases associated with percutaneous biliary drainage for extrahepatic cholangiocarcinoma. World J Gastroenterol. 2005;11:7024-7. 
21. Liu F, Li Y, Wei Y, Li B. Preoperative biliary drainage before resection for hilar cholangiocarcinoma: whether or not? A systematic review. Dig Dis Sci. 2011;56:663-72.

22. Jarnagin WR, Fong Y, DeMatteo RP, Gonen M, Burke EC, Bodniewicz BS, et al. Staging, resectability, and outcome in 225 patients with hilar cholangiocarcinoma. Ann Surg. 2001;234:507-17-9.

23. Matsuo K, Rocha FG, Ito K, D'Angelica MI, Allen PJ, Fong Y, et al. The Blumgart preoperative staging system for hilar cholangiocarcinoma: analysis of resectability and outcomes in 380 patients. J Am Coll Surg. 2012;215:343-55.

24. Lim JH, Choi GH, Choi SH, Kim KS, Choi JS, Lee WJ. Liver resection for Bismuth type I and type II hilar cholangiocarcinoma. World J Surg. 2013;37:829-37.

25. Song SC, Choi DW, Kow AW-C, Choi SH, Heo JS, Kim WS, et al. Surgical outcomes of 230 resected hilar cholangiocarcinoma in a single centre. ANZ J Surg. 2013;83:268-74.

26. Cannon RM, Brock G, Buell JF. Surgical resection for hilar cholangiocarcinoma: experience improves resectability. HPB (Oxford). 2012:14:142-9.

27. Hemming AW, Mekeel K, Khanna A, Baquerizo A, Kim RD. Portal vein resection in management of hilar cholangiocarcinoma. J Am Coll Surg. 2011;212:604-13

28. Tamoto E, Hirano S, Tsuchikawa T, Tanaka E, Miyamoto M, Matsumoto J, et al. Portal vein resection using the no-touch technique with a hepatectomy for hilar cholangiocarcinoma. HPB (Oxford). 2014;16:56-61.

29. Dumitrascu T, Chirita D, Ionescu M, Popescu I. Resection for hilar cholangiocarcinoma: analysis of prognostic factors and the impact of systemic inflammation on long-term outcome. J Gastrointest Surg. 2013; 17:913-24
30. Miyazaki M, Kato A, Ito H, Kimura F, Shimizu H, Ohtsuka M, et al. Combined vascular resection in operative resection for hilar cholangiocarcinoma: does it work or not? Surgery. 2007;141:581-8.

31. Kawasaki S, Imamura H, Kobayashi A, Noike T, Miwa S, Miyagawa S. Results of surgical resection for patients with hilar bile duct cancer. Ann Surg. 2003;238:84-92

32. Young AL, Prasad KR, Toogood GJ, Lodge JPA. Surgical treatment of hilar cholangiocarcinoma in a new era: comparison among leading Eastern and Western centers, Leeds. J Hepatobiliary Pancreat Sci. 2010;17:497-504.

33. Ratti F, Cipriani F, Ferla F, Catena M, Paganelli M, Aldrighetti LAM. Hilar cholangiocarcinoma: preoperative liver optimization with multidisciplinary approach. Toward a better outcome. World J Surg. 2013;37:1388-96.

34. Seyama Y, Kubota K, Sano K, Noie T, Takayama T, Kosuge T, et al. Long-term outcome of extended hemihepatectomy for hilar bile duct cancer with no mortality and high survival rate. Ann Surg. 2003;238:73-83.

35. Ito F, Agni R, Rettammel RJ, Been MJ, Cho CS, Mahvi DM, et al. Resection of hilar cholangiocarcinoma. Ann Surg. 2008;248:273-9.

36. Ramacciato G, Nigri G, Bellagamba R, Petrucciani N, Ravaioli M, Cescon $\mathrm{M}$, et al. Univariate and multivariate analysis of prognostic factors in the surgical treatment of hilar cholangiocarcinoma. Am Surg. 2010; 76:1260-8.

37. Dinant S, Gerhards MF, Busch ORC, Obertop H, Gouma DJ, Van Gulik TM. The importance of complete excision of the caudate lobe in resection of hilar cholangiocarcinoma. HPB (Oxford). 2005;7:263-7.

38. De Jong MC, Marques H, Clary BM, Bauer TW, Marsh JW, Ribero D, et al. The impact of portal vein resection on outcomes for hilar cholangiocarcinoma: a multi-institutional analysis of 305 cases. Cancer. 2012; 118:4737-47. 